\title{
LA LIBRE COMPETENCIA EN LA COMPRA PÚBLICA
}

\author{
FREE COMPETITION IN PUBLIC PROCUREMENT
}

\section{Juan David Dugue Botero}

Doctor en Derecho de la Universidad Alfonso X El Sabio (Madrid), magister en Derecho Administrativo de la Universidad del Rosario, experto en Contratos del Sector Público por la Universidad de Deusto

(Bilbao). Docente titular de la Facultad de Jurisprudencia de la Universidad del Rosario. ORCID: [https://orcid.org/0000-0002-5281-9793]. jduque@duquebotero.com

Recebido em: 04.02.2021
Aprovado em: 10.03.2021
DOI: [https://doi.org/10.48143/rdai/17.jddb]

ÁreA DO DIREITO: Administrativo

RESUMEN: El presente trabajo está dedicado a la investigación de la dinámica de la libre competencia en las compras públicas en el ordenamiento juridico colombiano. En esta propuesta, se delinea primero el marco legal de la libre competencia, para luego problematizar las dificultades de su implementación en el ámbito de la contratación pública. En este contexto, explica las prácticas anticompetitivas más perjudiciales para la libre competencia, la corrupción y la colusión, y presenta soluciones existentes para combatirlas, destacando a potestad sancionadora da Superintendencia de Industria y Comercio.

Palabras clave: Libre competencia - Compras públicas - Prácticas anticompetitivas - Corrupción - Colusión - Potestad sancionadora.

\begin{abstract}
This paper is dedicated to investigating the dynamics of free competition in public procurement in the Colombian legal system. In this proposal, firstly, outlines the legal framework of free competition, in order to problematize the difficulties of its implementation in the field of public procurement. In this context, it explains the most damaging anti-competitive practices for free competition, corruption and collusion, and presents the existing solutions to address them, highlighting the sanctioning power of the Superintendency of Industry and Commerce.
\end{abstract}

KeYwoRds: Free competition - Public purchases Anti-competitive practices - Corruption - Collusion - Sanctioning power.

SumÁrio: Libre competencia en la compra pública. Potestad sancionadora de la Superintendencia de Industria y Comercio. Bibliografía. 
una decisión sancionatoria o medida cautelar en este sentido, la Entidad podrá dar por terminado el contrato con fundamento en los artículos 44 y 45 de la Ley 80 de 1993.

Al respecto, la Corte Constitucional en sentencia T-1341 de 2001 estableció:

"Las causales de nulidad absoluta que permiten el ejercicio de la potestad excepcional contenida en el articulo 45 de la Ley 80 de 1993, a la cual se viene haciendo alusión, operan cuando la celebración del contrato estatal se efectúa con personas incursas en causales de inhabilidad o incompatibilidad previstas en la Constitución y la ley, o contra expresa prohibición constitucional o legal o cuando se declaren nulos los actos administrativos en que se fundamente el respectivo contrato. Esto significa que se refieren a situaciones de orden estrictamente jurídico que vician el contrato, afectando su validez jurídica e impidiendo que se inicie o se continue ejecutando" (Corte Constitucional, Sentencia T-1341, 2001).

En efecto, cuando la Entidad contratante observe el acaecimiento de alguno de los vicios establecidos en los numerales $1^{\circ}, 2^{\circ}$ y $4^{\circ}$ del artículo 44 de la Ley 80 de 1993, inmediatamente deberá declarar la terminación del contrato estatal y ordenar la liquidación de este, sin que sea necesario que exista declaración judicial al respecto, la cual podrá acaecer después.

Es menester recordar que todas las actuaciones que realice un servidor público se deben realizar bajo el debido proceso, razón por la cual sea cual sea la actuación, esta debe estar motivada, con los hechos claros y respetando la oportunidad de las partes de manifestarse, con el fin de salvaguardar los derechos de todos y la protección del interés general.

En conclusión, la contratación pública es un foco álgido de prácticas restrictivas de la competencia, donde la conducta de agentes que hacen parte de procesos de selección está al margen de lo consignado en el ordenamiento jurídico, afectando directamente el principio de selección objetiva, de igualdad, moralidad y buena fe. De lo expuesto se puede evidenciar que aún cuando el Estado destina los recursos correspondientes para contar con una infraestructura y personal capacitado que vigile, controle y sancione aquellas prácticas y agentes, las cifras anteriormente citadas sobre índices de corrupción no reflejan el esfuerzo del Estado.

\section{BIBLIOGRAFIA}

ACHILA PEÑALOSA, E. J. \& PABÓN ALMANZA, C. (2012). Colusión en Licitaciones y Concursos el caso paradigmático de las entidades públicas.

ALARCÓN PEÑA, Andrea (2016). "La libre competencia económica en el derecho colombiano: una revisión desde la Economía Social de Mercado y sus implicaciones normativas", en Revista Prolegómenos (37), pp. 109-124.

AROZAMENA, L., \& Weinschelbaum, F. (2010). Compras Públicas: Aspectos Conceptuales y Buenas Prácticas. Buenos Aires: Universidad Nacional de San Martín.

CONGRESO DE LA REPÚBLICA (1992). Exposición de Motivos Ley 80 de 1993. Gaceta del Congreso 75 del 23 de septiembre de 1992. 
CONGRESO DE LA REPÚBLICA (2007). Exposición de Motivos Proyecto de Ley 20 de 2005 Senado, por medio de la cual se introducen medidas para la eficiencia y la transparencia en la Ley 80 de 1993, y se dictan otras disposiciones generales sobre la contratación con recursos públicos. Gaceta del Congreso 458 del primero de agosto de 2005.

CORTE CONSTITUCIONAL. (24 de junio de 1991). Sentencia C-815. [MP Rodrigo Escobar Gil].

CORTE CONSTITUCIONAL. (8 de noviembre de 2007). Sentencia C-932. [MP Marco Gerardo Monroy Cabra].

CORTE CONSTITUCIONAL. (7 de octubre de 2009). Sentencia C-713. [MP María Victoria Calle Correa].

CORTE CONSTITUCIONAL. (11 de diciembre de 2001). Sentencia T-1341. [MP Álvaro Tafur Galvis].

OCDE (2009). Lineamientos para combatir la colusión entre oferentes en licitaciones públicas. Recuperado de: http://www.oecd.org/daf/competition/cartels/42761715.pdf Transparency International (29 de mayo de 2018). How do you define Corruption? Recuperado de: https://www.transparency.org/what-is-corruption\#define.

OECD (2016). Fighting bid rigging in public procurement: Report on implementing the OECD Recommendation. Recuperado de: http://www.oecd.org/daf/ competition/Fighting-bi cumple sólo con algunos de d-rigging-in-public-procurement-2016-implementation-report.pdf.

OSSA BOCANEGRA, C. E. (2014). Tratamiento de la colusión en la contratación pública: una visión del caso colombiano. Revista de Derecho, 42.

PALACIO JARAMILLO, M. T. (2002). Parámetros constitucionales para la determinación de un régimen jurídico de contratación pública en Colombia. Revista de derecho público Universidad de los Andes, 14.

SERRANO-PINILLA (2011). El derecho de la competencia como mecanismo para garantizar rivalidad en las licitaciones públicas e impulsar el crecimiento económico, 19. International Law, Revista Colombiana de Derecho Internacional.

SOTO PINEDA, J. A. (2014). La defensa de la competencia y el alcance de las ayudas públicas en el marco de los tratados de libre comercio. Vniversitas, 128.

SUPERINTENDENCIA DE INDUSTRIA Y COMERCIO (2010). Guía práctica para combatir la colusión en las licitaciones. Recuperado de: http://www.sic. gov.co/recursos_user/documentos/articulos/2010/Guia_Contratacion.pdf.

SUPERINTENDENCIA DE INDUSTRIA Y COMERCIO (2017). Protección de la Competencia, Beneficios por Colaboración. Recuperado de: http://www.sic. gov.co/beneficios-por-colaboracion.

SUPERINTENDENCIA DE INDUSTRIA Y COMERCIO (3 de septiembre de 2019) Resolución 42216 de 2019. Por la cual se imponen unas sanciones por infracciones al régimen de protección de la competencia. 
Superintendencia de Industria y Comercio (16 de septiembre de 2013) Resolución 54695 de 2013. Por la cual se imponen unas sanciones por infracciones al régimen de protección de la competencia.

TORRADO FRANCO, E. T. (2010). El régimen sancionatorio y el otorgamiento de garantías sobre prácticas restrictivas de la competencia en la Ley 1340 de 2009. Revista de Derecho Privado (43), 3-29.

TRANSPARENCIA POR COLOMBIA (2019). Colombia: entre los países donde esfuerzos anticorrupción están estancados.

VELANDIA, Mauricio, Derecho de la Competencia y del Consumo, Universidad Externado de Colombia. Bogotá 2008.

\section{Pesquisas do EDITORIAL}

\section{Veja também Doutrinas relacionadas ao tema}

- A promoção do desenvolvimento nacional sustentável como finalidade da licitação pública, de Silvia Portes Rocha Martins, Felipe José Ansaloni Barbosa e Sarah Carolina Zanetti e Viguetti - RT 993/329-446 (DTR|2018|17929);

- Discricionariedade administrativa e sustentabilidade, de José Américo Zampar Júnior e Juliana Carolina Frutuoso Bizarria - RT 1017/21-45 (DTR|2020|8178);

- La compra pública estratégica en España, de Alejandro Canónico Sarabia - RDAl 15/219-239 (DTR|2020|14269); e

- Os desafios para a implementação das licitações sustentáveis na Administração Pública, de Natália da Silveira Arenas - RDA 82/121-136 (DTR|2016|20505).

\section{Veja também Jurisprudência relacionada ao tema}

- Conteúdo exclusivo Web: JRP|2021/402275. 


\section{SUMÁRIO}

LINHA EDITORIAL - EDITORIAL LINE

APRESENTAÇÃO - INTRODUCTION

1. Seção - Direito Administrativo

Section 1 - Administrative LaW

A) Princíplos do Direito Administrativo

A) Principles of Administrative Law

Precedentes administrativos: um caminho para segurança jurídica nas relações entre a Administração Pública e os cidadãos

Administrative precedents: a path to legal security in the relationship between public administration and citizens

Carlos Henrique Benedito Nitão loureiro e Vladimir da Rocha França.....

Ética da discussão e princípios da administração pública: o administrador público mentiroso e a improbidade administrativa Discussion ethics and principles of public administration: the lying public administrator and administrative improbity

Caroline Müller Bitencourt e Janriê Rodrigues Reck.

B) Processo Administrativo

B) Administrative Proceedings

Consensualidade como modo alternativo de exercício da pretensão punitiva estatal no processo administrativo sancionador

Consensuality as an alternative mode for the exercise of state punitive pretension in the administrative sanctional process

Shirlei Silmara de Freitas Mello e Daniela Almeida Campos. 


\section{C) Licitação e Contratos Administrativos \\ C) Bidding and Administrative Contracts}

A consagração popular no procedimento de inexigibilidade para contratação de artistas musicais

The popular consecration in the inexigibility procedure for contracting musical artists

Pedro Henrique Maciel Barros e Marina Zava de Faria

D) Intervenção do Estado na Ordem Social

D) State Intervention in the Social Order

Defensoria Pública e legitimação social do direito: uma análise sobre reconhecimento e paridade participativa na tutela de grupos vulneráveis

Public Defender's office and social legitimation: an analysis on recognition and participative parity in the protection of vulnerable groups

Cristian Patric de Sousa Santos e Ricardo Maurício Freire Soares

\section{SeÇão - Complance}

\section{Section 2 - Compliance}

Compliance e responsabilidade de pessoas jurídicas

Compliance and responsibility of legal entities

Ricardo Marcondes Martins

Compliance ambiental-energético e a ecoeficiência empresarial: cenário na pandemia da Covid-19

Environmental-energy compliance and business eco-efficiency: pandemic scenario of Covid-19

Priscila Elise Alves Vasconcelos, Cleyson de Moraes Mello e Paulo Sérgio

VASCONCELOS

\section{SeÇÃo - Doutrina Estrangeira}

\section{SECTION 3 - FOREIGN DOCTRINE}

Sobre la naturaleza jurídica de los aeropuertos privados internacionales

The legal nature of international private airports

Jaime Rodriguez-Arana Muñoz 
La libre competencia en la compra pública

Free competition in public procurement

Juan David Duque Botero.

El procedimiento sancionadoren la Ley General de Responsabilidades Administrativas

The sanctioning procedure in the General Administrative Responsibilities Act

Manlio Fabio Casarin León.

\section{4a. SeÇão - Pareceres}

\section{Section 4 - Legal Opinions}

Controle do ato discricionário arbitrário na gradação da pena administrativa de multa aplicada pela administração pública federal, atecnia do caput do art. $2^{\circ}$ e inobservância da primariedade do inciso I, da Lei 7.889/1989: desrespeitos aos princípios da motivação, proporcionalidade, razoabilidade e direitos fundamentais da empresa/administrada que pode inviabilizar atividade econômica de empresa de economia familiar protegida pelo artigo 170 da Constituição de 1988

Control of the arbitrary discretionary act in grading the fine administrative penalty applied by the federal public administration, atecnia of the caput of art. 2nd and inobservance of the primariety of item I, of Law 7,889/1989: disrespect to the principles of motivation, proportionality, reasonability and fundamental rights of the company/manager that may invibialize the economic activity of the family economy company protected by article 170 of the 1988 Constitution

Jerson Carneiro Gonçalves Junior

\section{SeÇÃo - ComentÁrios À JURISPRUdÊnCIA \\ SECTION 5 - CommentS of JuRISPRUdENCE}

ADI 6.427 DF: inovação normativa pelo Judiciário na interpretação conforme a Constituição

ADI 6,427 DF: normative innovation by the Judiciary in the interpretation according to the Constitution

Adilson Neri Pereira 
Acórdão 169/2021-TCU: um novo entendimento jurisprudencial para o cálculo da garantia adicional em contratos administrativos Judgment 169/2021-TCU: a new jurisprudential understanding for the calculation of the additional guarantee in administrative contracts

Augusto César Tavares de Lira da Cunha e Pedro Flávio Cardoso lucena .... 301

\section{SeÇÃo - EntreVISTA}

\section{Section 6 - InTERVIEW}

Entrevista com Dalmo de Abreu Dallari Interview with Dalmo de Abreu Dallari

Augusto Neves Dal Pozzo e Ricardo Marcondes Martins.

\section{Seção - Memória do Direito Administrativo}

\section{Section 7 - Retrospective of Administrative Law}

0 Conselho de Estado e o contencioso administrativo no Brasil The Council of State and administrative litigation in Brazil

Modalidades de descentralização administrativa e seu controle Modalities for administrative decentralization and its control

Celso Antônio Bandeira de Mello.

Impossibilidade de o INPS multar municípios Impossibility for the Brazilian Institute of Social Welfare to fine municipalities

Celso Antônio Bandeira de Mello.

A personalidade do Estado

The personality of the State

Oswaldo Aranha Bandeira de Mello.

Normas de Publicação para Autores de Colaboração Autoral Inédita ..... 393

Publication Guidelines for Authors to Submit Unpublished Articles ...... 395 




\section{NESTA EDIÇÃO:}

\section{ÉTICA DA DISCUSSÃO E PRINCÍPIOS DA ADMINISTRAÇÃO PÚBLICA: O ADMINISTRADOR PÚBLICO MENTIROSO E A IMPROBIDADE ADMINISTRATIVA}

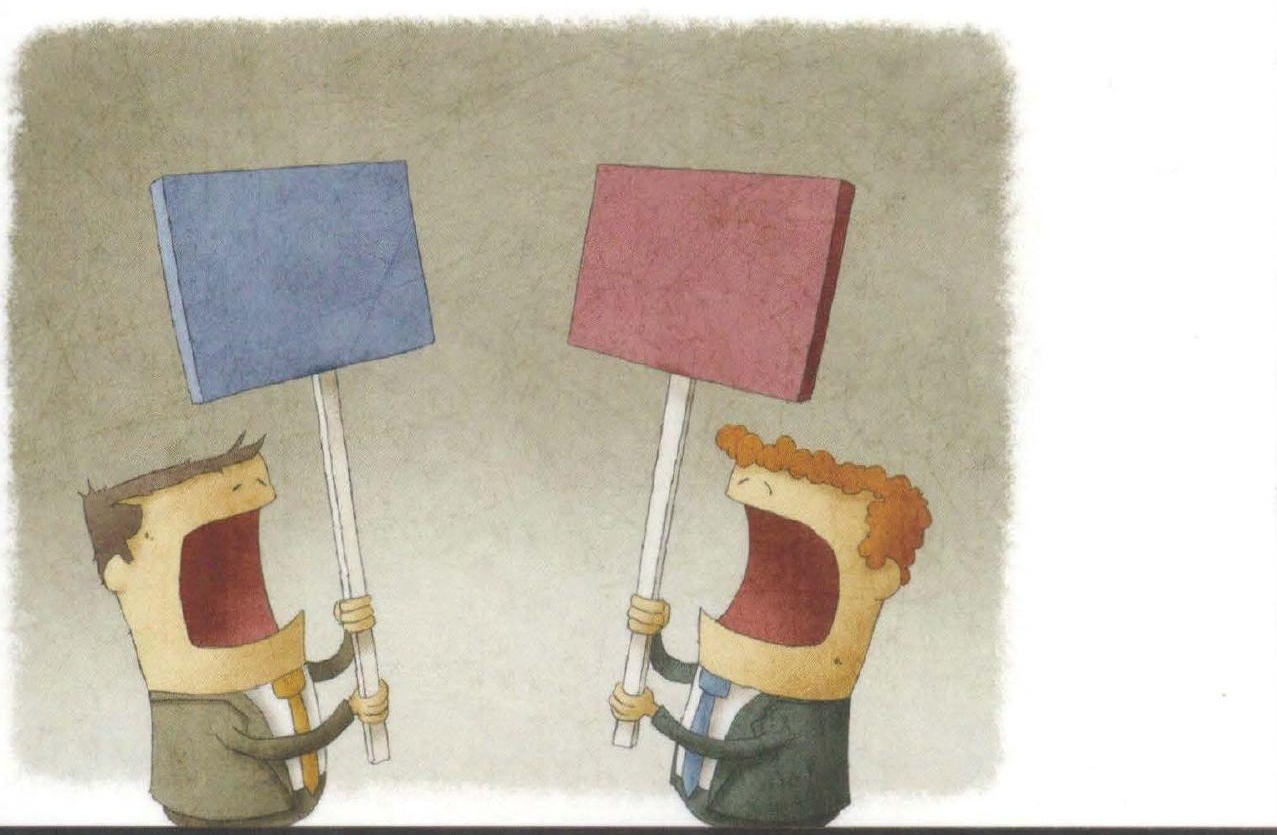

REVISTA DE DIREITO ADMINISTRATIVO, INFRAESTRUTURA, REGULAÇÃO E COMPLIANCE
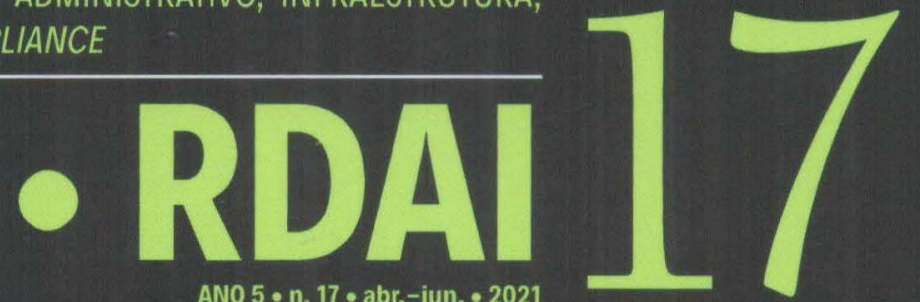

Journal of Administrative Law, Infrastructure, Regulation and Compliance

N. 5 • ISSUE $17 \cdot$ April - June $\cdot 2021$

COORDENACẢO

AUGUSTO NEVES DAL POZZO E RICARDO MARCONDES MARTINS 